\title{
Gnevyshev peaks in solar radio emissions at different frequencies
}

\author{
R. P. Kane \\ Instituto Nacional de Pesquisas Espacias, C.P. 515 São Jose dos Campos, 12201-970, SP, Brazil \\ Received: 13 October 2008 - Revised: 6 February 2009 - Accepted: 27 February 2009 - Published: 1 April 2009
}

\begin{abstract}
Sunspots have a major 11-year cycle, but the years near the sunspot maximum show two or more peaks called GP (Gnevyshev Peaks). In this communication, it was examined whether these peaks in sunspots are reflected in other parameters such as Lyman- $\alpha$ (the chromospheric emission $121.6 \mathrm{~nm}$ ), radio emissions $242-15400 \mathrm{MHz}$ emanating from altitude levels $2000-12000 \mathrm{~km}$, the low latitude $\left(+45^{\circ}\right.$ to $-45^{\circ}$ ) solar open magnetic flux and the coronal green line emission (Fe XIV, $530.3 \mathrm{~nm}$ ). In the different solar cycles 20-23, the similarity extended at least upto the level of $609 \mathrm{MHz}$, but in cycle 22, the highest level was of $242 \mathrm{MHz}$. The extension to the higher level in cycle 22 does not seem to be related to the cycle strength $R z(\max )$, or to the cycle length.
\end{abstract}

Keywords. Solar physics, astrophysics, and astronomy (Corona and transition region; Photosphere and chromosphere; Radio emissions)

\section{Introduction}

Sunspots have a major 11-year cycle. However, the sunspot maximum is not smooth but structured. Two or more peaks can be identified during the sunspot maximum years. This splitting of activity was identified for the first time in the green corona line intensity data by Gnevyshev $(1967,1977)$ and later for several solar and interplanetary phenomena (details in the review by Storini et al., 2003). These will be termed henceforth as GPs (Gnevyshev Peaks). In recent publications (Kane, 2006, 2007, 2008a, b, c), it was pointed out that these GPs have a solar latitude dependence, with peaks shifting with time from higher to lower latitudes as in the Maunder butterfly diagram, and the peaks in sunspots are similar to those in other electromagnetic ra-

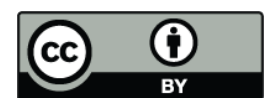

Correspondence to: R. P. Kane (kane@dge.inpe.br) diations (2800 MHz flux, X-ray background etc.), but differ in number and time location for some other parameters like coronal mass ejections (CMEs), solar open magnetic flux etc.

Upto what altitudes does the similarity of sunspot GPs prevail? The $2800 \mathrm{MHz}(10.7 \mathrm{~cm})$ flux (originating approximtely in lower corona) shows GPs similar to sunspots. However, data are available for other solar radio emissions also, at several other frequencis. Do these also show a similar behaviour? The Sun emits radio energy with a slowly varying intensity. The Radio flux originates from atmospheric layers high in the Sun's chromosphere and low in its corona, and changes gradually from day to day, in response to the number of spot groups on the disk. Radio intensity levels consist of emission from three sources, namely, from the undisturbed solar surface, from developing active regions, and from short-lived enhancements above the daily level (detailed description in NOAA website ftp://ftp.ngdc.noaa.gov/ STP/SOLAR_DATA/SOLAR_RADIO/FLUX/read.me). Radio emissions can be due to several mechanisms (detailed description of mechanisms etc. is given in Kane, 2004). Models of plasma density and radio emissions give approximate heights for the various emissions. Aschwanden and Benz (1995) and Melendez et al. (1999) have discussed a model which sets the density at different heights above the solar surface. From this model, the plasma frequency at different heights can be calculated.. In general, higher frequencies can be assumed to be able to emerge from deeper regions (lower solar altitudes). The temperature-height profiles are given in Fontenla et al. (1999), where it is mentioned that (1) the temperature drops from $\sim 6000 \mathrm{~K}$ at the solar surface to $\sim 4800 \mathrm{~K}$ at $500 \mathrm{~km},(2)$ then rises to $6000 \mathrm{~K}$ at $900 \mathrm{~km}, 8000 \mathrm{~K}$ at $1900 \mathrm{~km}, 10000 \mathrm{~K}$ at $2100 \mathrm{~km}$, (3) increases rapidly to $\sim 500000 \mathrm{~K}$ in a narrow transition region around $2100 \mathrm{~km}$, (4) reaches $\sim 900000 \mathrm{~K}$ at $2800 \mathrm{~km}$, and a million degrees or more in the corona. The absolute altitude levels from which radio frequencies escape are uncertain and all height estimates are very approximate. However, it is assumed that in a relative way, higher frequencies would

Published by Copernicus Publications on behalf of the European Geosciences Union. 


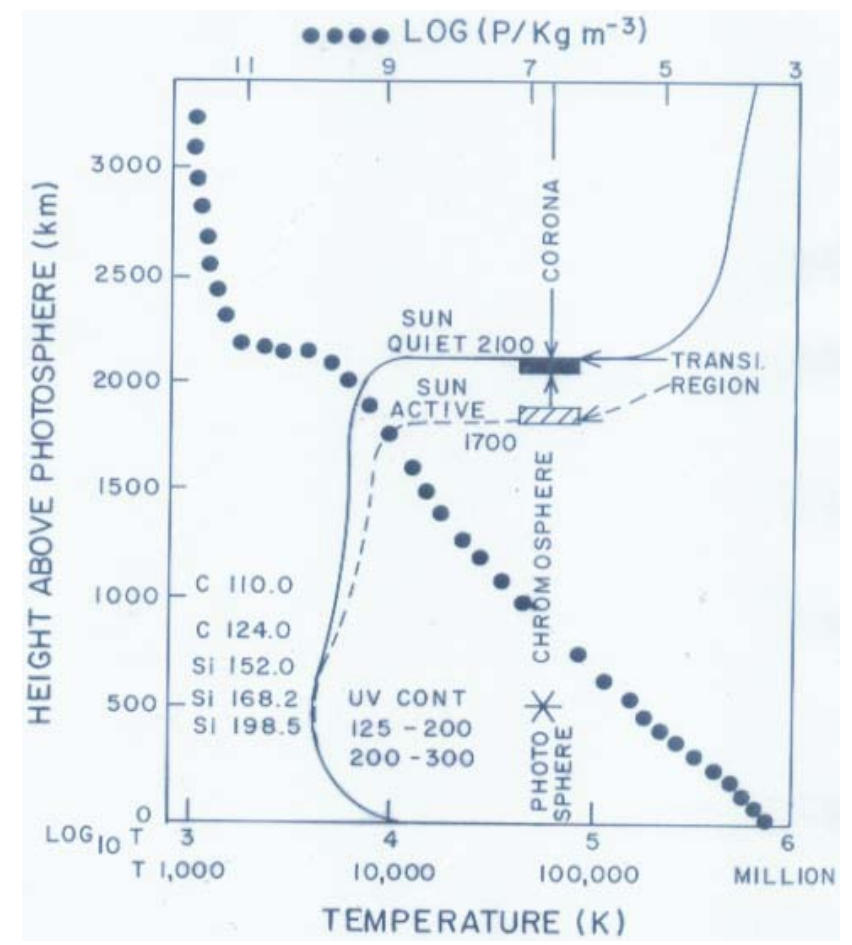

Fig. 1. Plot of height above the photosphere (ordinate, $\mathrm{km}$ ) versus temperature (K) (abscissa, bottom) and plasma density $P$ (abscissa, top; note the reversed scale for $P$, increasing from right to the left)).

escape from lower depths (lower solar altitudes); so the relative comparison should be qualitatively valid, paticularly when data used are averages over long time intervals (not hours or days, but months). The flux range $275-15000 \mathrm{MHz}$ is most probably from the upper corona down to the upper chromosphere.

In this communication, medium-term variations (peaks separated by a few months during $\sim 6$ years near sunspot maximum) are examined and compared with sunspot variation, for different radio frequencies for solar cycles 20-23.

\section{Data}

Solar flux density at $2800 \mathrm{MHz}(10.7 \mathrm{~cm})$ had been recorded routinely by the radio telescope near Ottawa, Canada since 14 February 1947. Beginning in June 1991, the solar flux density measurement source is Penticton, B.C., Canada. Besides the $2800 \mathrm{MHz}$ flux, several frequencies are recorded at several other locations, (website ftp://ftp.ngdc.noaa.gov/STP/SOLAR_DATA/ SOLAR_RADIO/FLUX//USAF_NOON_FLUX).

Data from May 1966 through December 1987 are from Sagamore Hill (SGMR) in Massachusetts only. From 1988 onwards, data are available from Palehua (PALE), Hawaii, San Vito (SVTO), Italy, Learmonth (LEAR), Australia, and Sagamore Hill. Data were averaged for all these locations for

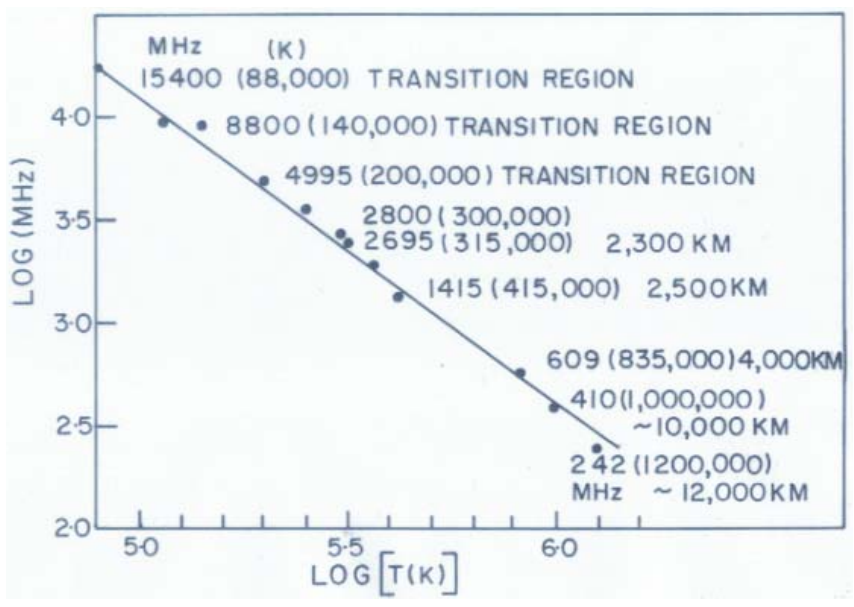

Fig. 2. Plot of radio frequencies $\mathrm{MHz}$ (ordinate) versus temperature $\mathrm{T}(\mathrm{K})$ (abscissa). Since ranges were very wide $(\sim 200-17000 \mathrm{MHz}$, and $88000-1200000 \mathrm{~T}(\mathrm{~K})$ ), the plots are logarithmic, but the actual values are marked near the relevant points (e.g., $15400 \mathrm{MHz}$, $\mathrm{T}(\mathrm{K}) 88000 \mathrm{~K}$, altitude TRANSITION REGION; $410 \mathrm{MHz}, \mathrm{T}(\mathrm{K})$ 1000000 , altitude $\sim 12000 \mathrm{~km}$ ).

the following frequencies, 242, 410, 609, 1415, 2695, 4995, 8800 , and $15400 \mathrm{MHz}$ (besides $2800 \mathrm{MHz}$ ).

\section{Characteristics of solar atmosphere}

Figure 1 shows the plot of the height above the photosphere (ordinate, $\mathrm{km}$ ) versus temperature (K) (abscissa, bottom) and plasma density $P$ (abscissa, top). The temperature in the very thin $(300 \mathrm{~km})$ transition region changes rapidly from $\sim 10000 \mathrm{~K}$ to $\sim 120000 \mathrm{~K}$. Above $\sim 2100 \mathrm{~km}$, the temperature rises to million $\mathrm{K}$ or more and the height-temperature relationship above $3500 \mathrm{~km}$ is not very certain; but even at $3500 \mathrm{~km}$ (upper limit of this plot), the temperature is already several hundreds of thousands $\mathrm{K}$. The plasma density $P$ (note the reversed scale at the top, increasing from right to left so that the $P$ trace, big full dots, does not mix up with the temperature trace, lines)) is highest near the photosphere (temperature $\sim 6000 \mathrm{~K}$ ) and decreases rapidly in the high temperature regions at higher altitudes. The implication of this for solar radio emissions is shown in Fig. 2. Since the ranges are very wide $(\sim 200-17000 \mathrm{MHz}$, and $\sim 88000$ $1200000 \mathrm{~T}(\mathrm{~K})$ ), the plots are logarithmic, but the actual values are marked near the relevant points (e.g., $15400 \mathrm{MHz}$, $\mathrm{T}(\mathrm{K}) 88000 \mathrm{~K}$, altitude TRANSITION REGION; $410 \mathrm{MHz}$, $\mathrm{T}(\mathrm{K}) 1000000$, altitude $\sim 12000 \mathrm{~km}$ ).

\section{Plots of some solar parameters, including solar radio emissions}

Radio emissions have contributions from short-lived bursts (minutes to hours) as well as day-to-day variations. As the 


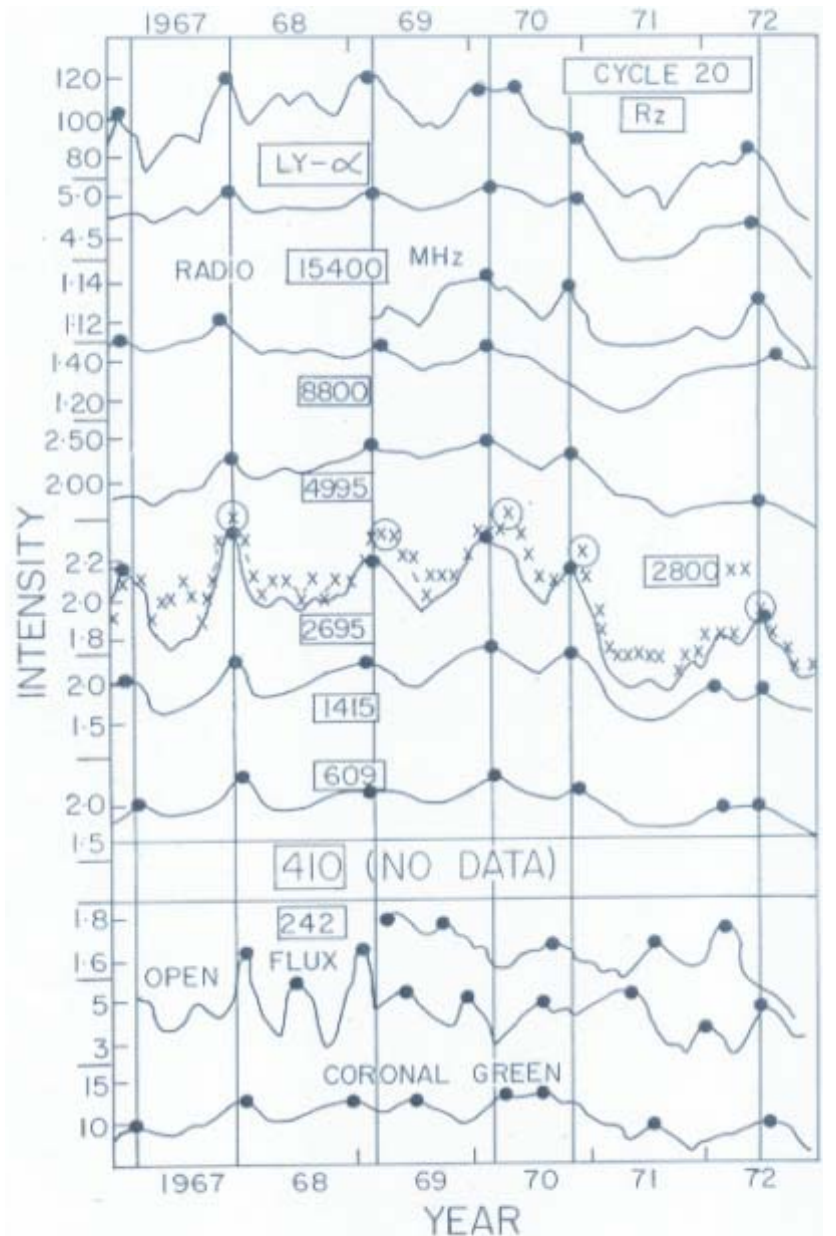

Fig. 3. Plots of 3-month moving averages during 1967-1972, peak years of solar cycle 20. First (top) plot is for sunspot number $R z$. Second plot is for Lyman- $\alpha(121.6 \mathrm{~nm})$. Further plots are for radio emissions 15 400, 8800, 4995, 2695 (2800 as superposed crosses), $1415,609,410$ (data missing) and $242 \mathrm{MHz}$. More than three peaks are marked by vertical lines). The second plot from bottom is for the solar open magnetic flux at low solar latitudes $+45^{\circ}$ to $-45^{\circ}$. The bottom plot is for the coronal green line emission (Fe XIV, $530.3 \mathrm{~nm}$ ).

purpose was to study medium- and longterm variations (few months), abnormal daily values due to solar flare need to be deleted. This seems to have already been done in the published data, but as a further precaution, we deleted daily values deviating by more than $30 \%$ from the monthly mean. Thus, values deviating by $30 \%$ or less were retained and the error due to these in the monthly mean could be still $\sim 1 \%$. Monthly values were freshly recalculated, and these were smoothed further by calculating 3-month moving averages. These are certainly not expected to have any influence of individual day solar flares by more than a few fractions of $1 \%$, which would be negligible compared to the magnitude of the effects studied, about 10-20\% from a Gnevyshev peak

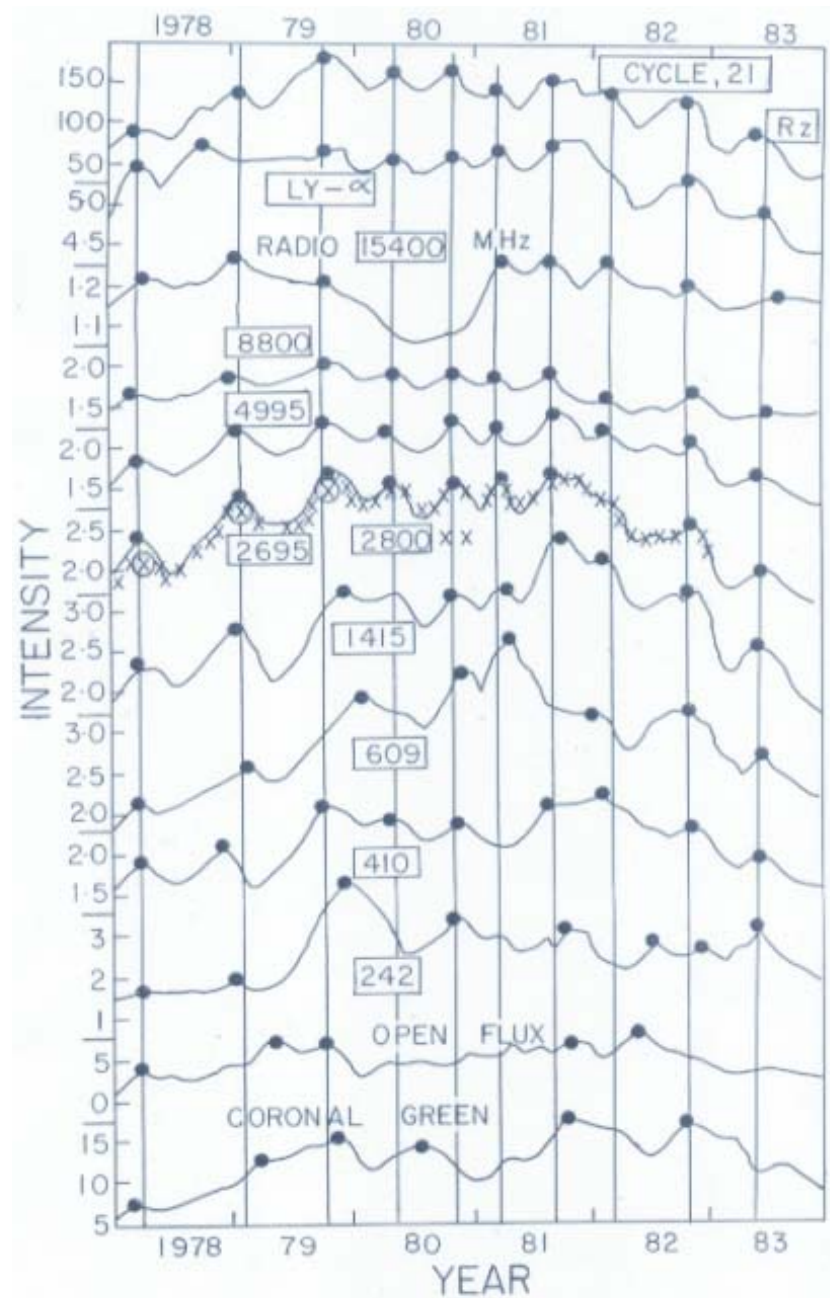

Fig. 4. Same as Fig. 3, but for 1978-1983, peak years of solar cycle 21 .

to a Gnevyshev gap. Figure 3 shows the plots of 3-month moving averages during 1967-1972, peak years of solar cycle 20. The first (top) plot is for sunspot number $R z$. Several peaks are seen (marked by full dots, every peak defined as a monthly value larger than two previous and two succeeding monthly values). The second plot is for Lyman- $\alpha$, a chromospheric emission $(121.6 \mathrm{~nm})$. The peaks are marked by full dots and tally with those of the sunspots. Further plots are for radio emissions 15 400, 8800, 4995, 2695 (2800 as superposed crosses), 1415, 609, 410 (data missing) and $242 \mathrm{MHz}$. The peaks marked with full dots almost tally with those of sunspots (for easy comparison, peaks tallying in more than three parameters, are marked by vertical lines) up to $609 \mathrm{MHz}$. Data for $410 \mathrm{MHz}$ are missing during this cycle 20, but the plot for $242 \mathrm{MHz}$ further down (starting only in 1969) shows peaks dissimilar to those of other parameters above. The second plot from bottom is for the solar open magnetic flux at low solar latitudes $+45^{\circ}$ to $-45^{\circ}$ 


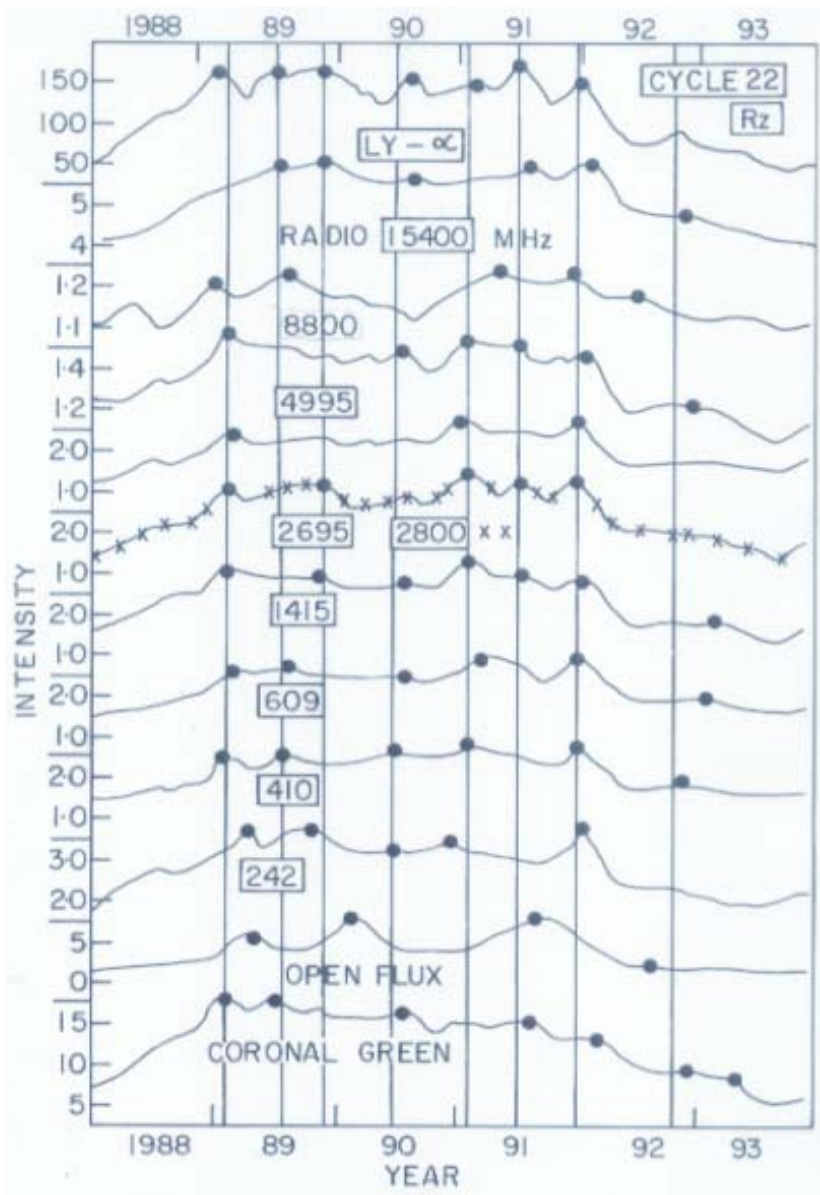

Fig. 5. Same as Fig. 3, but for 1988-1993, peak years of solar cycle 22 .

(Wang and Sheeley, 2002; Wang et al., 2000), estimated at $\sim 20$ solar radii. There are peaks but these differ considerably from those of sunspots etc. Finally, the bottom plot is for the coronal green line emission (Fe XIV, $530.3 \mathrm{~nm}$ ). Here, two peaks tally with sunspot peaks but many others do not tally. Thus, the similarity with sunspots peaks (matching within one month) seems to extend to the level from which $609 \mathrm{MHz}$ can escape (temperature region $\sim 835000 \mathrm{~K}$, altitude $\sim 4000 \mathrm{~km}$ above the photosphere). For higher regions, there are dissimilarities.

Figure 4 shows similar plots for 1978-1983, peak years of solar cycle 21 . Here, good matching seems to extend only up to the level of 2695 (or 2800) MHz (temperature $~ 300000 \mathrm{~K}$, altitude $\sim 2300 \mathrm{~km}$ ). For higher altitudes, one or more peaks show a displacement of more than one month from the vertical lines.

Figure 5 shows similar plots for 1988-1993, peak years of solar cycle 22. Here, good matching seems to extend to the level of $410 \mathrm{MHz}$ (temperature $\sim 1000000 \mathrm{~K}$, altitude $\sim 10000 \mathrm{~km}$ ). For higher altitudes, one or more peaks show a displacement of more than one month from the vertical lines.

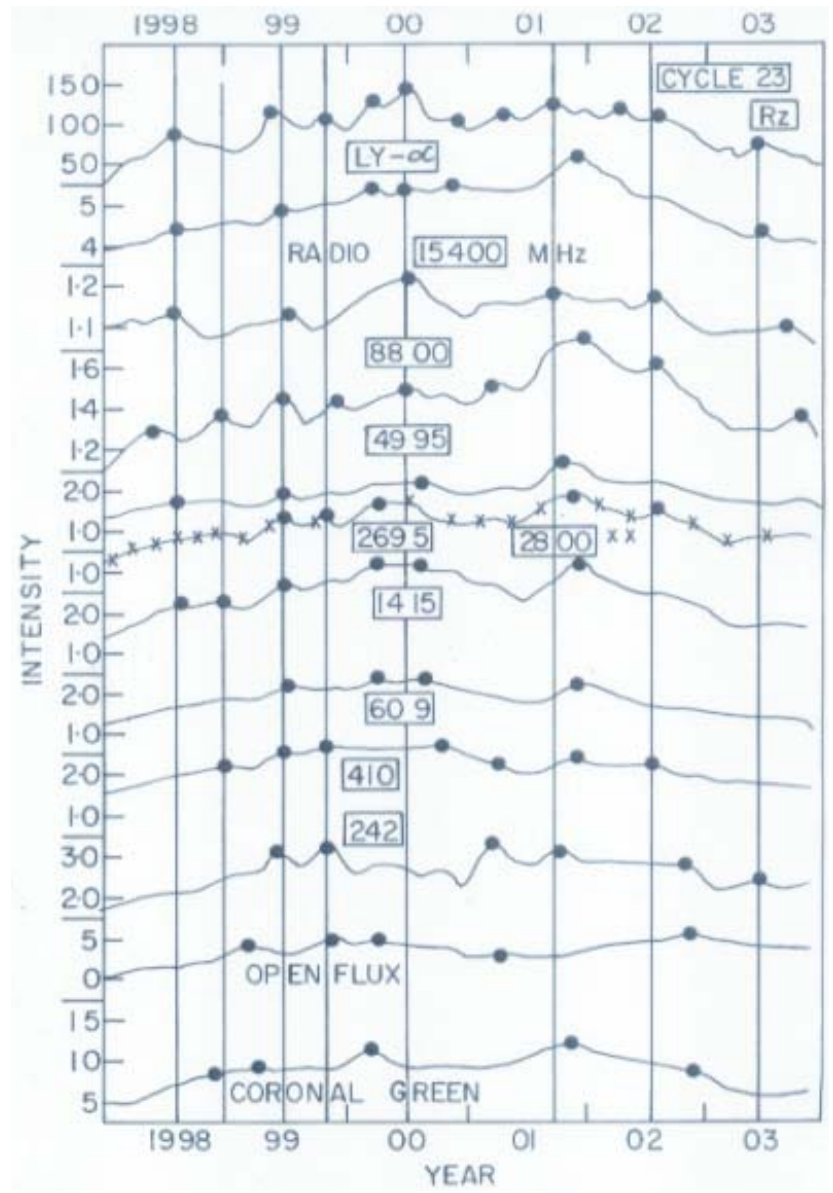

Fig. 6. Same as Fig. 3, but for 1998-2003, peak years of solar cycle 23 .

Figure 6 shows similar plots for 1998-2003, peak years of solar cycle 23. Here, there are many peaks in sunspots, and some of these show good matching only up to the level of $4.995 \mathrm{MHz}$, probably upto $\sim 2500 \mathrm{MHz}$ to a lesser extent, ((temperature $\sim 300000 \mathrm{~K}$, altitude $\sim 2300 \mathrm{~km}$ ). For higher altitudes, one or more peaks show a displacement of more than one month from the vertical lines.

To get a quantitative measure of the relationship between sunspot numbers and the radio frequencies, correlations were calculated for 48 months near sunspot maximum in each cycle. Years chosen were 1968-1971 for cycle 20, 1979-1982 for cycle 21, 1989-1992 for cycle 22 and 1999-2002 for cycle 23. Table 1 shows the values. The following may be noted:

1. For $15400 \mathrm{MHz}$, level 1, which is in the lower part of the transition region $(\sim 2000 \mathrm{~km}$ solar altitude), the correlation with sunspots is moderate $(0.60)$ in cycle 22 and $\operatorname{good}(0.94,0.82)$ in cycles 20,23 , but in cycle 21 , the correlation is very low, probably because of shorter and unreliable data. In general, one would say that the 
Table 1. Correlations between the 48 simultaneous monthly values ( 4 years) of sunspot number $R z$ versus the radio frequencies at solar altitude levels $1-8$ and coronal green line index. Standard errors for correlations above 0.70 are \pm 0.07 or less.

\begin{tabular}{|c|c|c|c|c|c|c|c|c|c|c|c|c|}
\hline \multicolumn{3}{|c|}{ Level } & 1 & 2 & 3 & 4 & 5 & 6 & 7 & 8 & 9 & 10 \\
\hline \multirow{2}{*}{\multicolumn{3}{|c|}{$\begin{array}{c}\text { Solar altitude }(\mathrm{km}) \text { approx. } \\
\text { Frequency MHz }\end{array}$}} & 2000 & 2100 & 2200 & 2300 & 2500 & 4000 & 10000 & 12000 & corona & cycle \\
\hline & & & 15400 & 8800 & 4995 & 2695 & 1415 & 609 & 410 & 242 & green & length \\
\hline 20 & 1968-1971 & 111 & 0.94 & 0.91 & 0.92 & 0.96 & 0.83 & 0.94 & No data & 0.71 & 0.89 & 138 \\
\hline 21 & 1979-1982 & 165 & -0.35 & 0.92 & 0.89 & 0.94 & 0.01 & 0.19 & 0.25 & 0.67 & -0.41 & 124 \\
\hline 22 & 1989-1992 & 159 & 0.60 & 0.98 & 0.85 & 0.92 & 0.97 & 0.79 & 0.88 & 0.96 & 0.98 & 115 \\
\hline 23 & 1999-2002 & 120 & 0.82 & 0.46 & 0.71 & 0.79 & 0.98 & 0.72 & 0.44 & -0.19 & 0.71 & 146 \\
\hline
\end{tabular}

Genvyshev peak structure in sunspot numbers did reflect in level 1.

2. For the $8800 \mathrm{MHz}$, level 2 in transition region ( $\sim 2100 \mathrm{~km}$ solar altitude), the correlations are very good (above 0.90 ) in cycles 20,21, 22 but moderate $(0.46)$ in cycle 23 . This is mainly because as seen in Fig. 6, though the peak matching between sunspot number and $8800 \mathrm{MHz}$ is reasonably good, the $8800 \mathrm{MHz}$ plot shows an abnormal increase during 2001-2002, while the sunspots have no such increase. Thus, just peak matching will not ensure good correlation unless the overall trend during the 48 months is also similar (it is mostly flat for sunspot numbers).

3. For the $4995 \mathrm{MHz}$, level 3 in the transition region $(\sim 2200 \mathrm{~km}$ solar altitude), correlations are good (above $0.70)$ for all cycles. So, level 3 has a good match with sunspot number Gnevyshev peaks.

4. For the $2695 \mathrm{MHz}$ (also $2800 \mathrm{MHz}$ ), level 4, $\sim 2300 \mathrm{~km}$ solar altitude, correlations are good (above 0.78 ) for all cycles. So, level 4 has a good match with sunspot number Gnevyshev peaks.

5. For $1415 \mathrm{MHz}$, level $5, \sim 2500 \mathrm{~km}$ solar altitude, correlations are good (above 0.78) for cycles 20, 22, 23, but for cycle 21 , the correlation is almost zero. This is because there is a strong uptrend in the $1415 \mathrm{MHz}$ plot, in contrast to a steady level for sunspots. So, level 4 has, in general, a good match with sunspot number Gnevyshev peaks.

6. For $609 \mathrm{MHz}$, level 6, $\sim 4000 \mathrm{~km}$ solar altitude, correlations are good (above 0.72) for cycles 20, 22, 23, but for cycle 21 , the correlation is very low (0.19) This is because there is a strong uptrend in the $609 \mathrm{MHz}$ plot, in contrast to a steady level for sunspots. So, level 6 has, in general, a good match with sunspot number Gnevyshev peaks.

7. For $410 \mathrm{MHz}$, level $7, \sim 10000 \mathrm{~km}$ solar altitude, correlation is good $(0.88)$ only for cycle 22 . For cycle 20 , there are no data. For cycles 21, 23, the correlations are poor or moderate. In cycle 21 , there was a big hump in $410 \mathrm{MHz}$ plot during 1979-1980, and some peaks did not match. This spoiled the correlation. In cycle 23, there were no trends in $410 \mathrm{MHz}$, but some peaks did not match. So, pnly moderate correlation (0.44) was seen. So, level 7 has, in general, a poor match with sunspot number Gnevyshev peaks.

8. For $242 \mathrm{MHz}$, level $8, \sim 12000 \mathrm{~km}$ solar altitude, for cycles 20,22 , the correlations are good $(0.71,0.96)$ for cycles 20,22 , moderate $(0.67)$ for cycle 21 and very poor $(-0.19)$ for cycle 23 , mostly because of mismatch of peaks. So, level 8 has, in general, a good match with sunspot number Gnevyshev peaks.

9. Summarizing, all the levels 1-6 have a good match between sunspots and radio frequencies up to $609 \mathrm{MHz}$. Above that (higher levels 7 and 8), the matching is poor for the odd cycles 21 and 23 and good for the even cycles 20,22 . Since the sunspot maxima $R z(\max )$ for these are high as well as low, a dependence on the strength of the sunspot cycle does not seem to be involved. The cycle lenghts for 21 and 23 are also low (124) as well as high (>146). Thus, a dependence on the lenghts of the sunspot cycle also, does not seem to be involved. Incidentally, the coronal green line index (level 9) shows correlation similar to $609 \mathrm{MHz}$, level 9, except for cycle 21 where the correlation is poor, mainly because there is an uptrend in the coronal index but not in sunspot number.

10. The coronal green line index shows correlations similar to those for $609-1415 \mathrm{MHz}$.

11. From the 35 correlations. ( 4 cycles, 8 radio frequencies and 1 coronal index, one value missing), 25 were good (above 0.70), 6 were poor (below 0.30, including negative) and 4 were moderate $(0.40-0.69)$. From these, some in cycle 21 had problems of abnormal up trends. If the linear trends were removed, the correlations increased substatially. For example, in case of $1415 \mathrm{MHz}$ in cycle 21 , the correlation increased from almost zero 
to 0.89 , and for $609 \mathrm{MHz}$, the value increased from 0.19 to 0.78 . Thus, peak matching was there but it was camouflaged by the radio frequency intensity uptrends, which did not exist in sunspot numbers.

12. In two cases, $15400 \mathrm{MHz}$ and $410 \mathrm{MHz}$ in cycle 21 , the correlations were low because of abnormal bumps or troughs in radio intensities, which did not exist in sunspot numbers.

\section{Conclusions and discussions}

Sunspots have a major 11-year cycle, but on fine time scale (months), the years near the maximum show two or more peaks called GP (Gnevyshev Peaks, Gnevyshev, 1967, 1977). In this communication, it was examined whether these peaks in sunspots are reflected in other parameters such as Lyman- $\alpha$ (the chromospheric emission $121.6 \mathrm{~nm}$ ), radio emissions $242-15400 \mathrm{MHz}$ emanating from altitude levels $2000-12000 \mathrm{~km}$, the low latitude $\left(+45^{\circ}\right.$ to $\left.-45^{\circ}\right)$ solar open magnetic flux (Wang and Sheeley, 2002; Wang et al., 2000), estimated at $\sim 20$ solar radii, and the coronal green line emission (Fe XIV, $530.3 \mathrm{~nm}$ ).

1. For 1968-1971, peak years of solar cycle 20, the similarity with sunspots (matching within one month) extended to the level from which $609 \mathrm{MHz}$ can escape (temperature region $\sim 835000 \mathrm{~K}$, altitude $\sim 4000 \mathrm{~km}$ above the photosphere). For higher regions, the similarity was doubtful.

2. For 1979-1982, peak years of solar cycle 21, the similarity extended only up to the level of 2695 (or 2800 ) $\mathrm{MHz}$ (temperature $\sim 300000 \mathrm{~K}$, altitude $\sim 2300 \mathrm{~km}$ ). For higher altitudes, similarity was doubtful.

3. For 1989-1992, peak years of solar cycle 22, the similarity extended up to the level of $242 \mathrm{MHz}$ (temperature $\sim 1200000 \mathrm{~K}$, altitude $\sim 12000 \mathrm{~km}$ ).

4. For 1999-2002, peak years of solar cycle 23, the similarity extended up to the level of $609 \mathrm{MHz}$. For higher altitudes, similarity was not seen.

Thus, in the different solar cycles 20-23, the similarity extended to the level of $609 \mathrm{MHz}$ certainly but to the highest level of $242 \mathrm{MHz}$ in cycle 22. This extension to the higher level in cycle 22 does not seem to be related to the cycle strength $R z$ (max), or to the cycle length. A possible reason for the differences could be that the force with which the solar photospheric dynamical upheavals is pushed upward could be different for different cycles. However, a correlation with the magnitude $R z(\max )$ etc. was not seen. Obviously, some other factors not considered here are the pushing forces. This needs further exploration.
Though peak timings are alike in the similarity region, there are some quantitative discrepancies. These have been presented in detail in earlier publications Kane (2002, 2004), but some glaring ones are pointed out here again. Thus, during 1967-1972 (Fig. 3), whereas three successive peaks in sunspots and Lyman- $\alpha$ have almost the same intensity, similar peaks in the $8800 \mathrm{MHz}$ plot are in descending order, while those in 4995 and $1495 \mathrm{MHz}$ are in ascending order. During 1978-1983 (Fig. 4), 4995 and $1495 \mathrm{MHz}$ show a large ascending tendency unlike other parameters. During 1998-2003 (Fig. 6), $8800 \mathrm{MHz}$ shows an abnormal ascending tendency, unlike others. Thus, some frequencies show behaviour very.different from others. Unless data errors are invoked (doubtful), perhaps here is some information relevant for modelling the processes which cause the radio emissions and their time variation. This too needs further exploration.

Acknowledgements. This work was partially supported by FNDCT, Brazil, under contract FINEP-537/CT.

Topical Editor R. Forsyth thanks two anonymous referees for their help in evaluating this paper.

\section{References}

Aschwanden, M. J. and Benz, A. O.: Chromospheric Evaporation and Decimetric Radio Emission in Solar Flares, Astrophys. J., 438, 997-1012, 1995.

Fontenla, J., White, O. R., Fox, P. A., Avrett, E. H., and Kurucz, R. L.: Calculation of Solar irradiances. I. Synthesis of the Solar spectrum, Astrophys. J., 518, 480-499, 1999.

Gnevyshev, M. N.: On the 11-Years Cycle of Solar Activity, Solar Phys., 1, 107-120, 1967.

Gnevyshev, M. N.: Essential features of the 11-year solar cycle, Solar Phys., 51, 175-183, 1977.

Kane, R. P.: Evolutions of various solar indices around sunspot maximum and sunspot minimum years, Ann. Geophys., 20, 741755, 2002,

http://www.ann-geophys.net/20/741/2002/.

Kane, R. P.: Long-Term and Medium-Term Variations of Solar Radio Emissions at Different frequencies, Solar Phys., 219, 357365, 2004.

Kane, R. P.: Comparison of the variations of CMEs and ICMEs with those of other solar and interplanetary parameters during solar cycle 23, Solar Phys., 233, 107-115, 2006.

Kane, R. P.: Latitude Dependence of the 'Gnevyshev' Peaks and Gaps, Solar Phys., 245, 415-421, 2007.

Kane, R. P.: Similarities and Dissimilarities between the Variations of CME and Other Solar Parameters at Different Heliographic Latitudes and Time Scales, Solar Phys., 248, 177-190, 2008 a.

Kane, R. P.: Latitude dependence of the variations of sunspot group numbers (SGN) and coronal mass ejections (CMEs) in cycle 23, Solar Phys., 249, 355-367, 2008b.

Kane, R. P.: Gnevyshev peaks and gaps for Coronal Mass Ejections (CMEs) of different widths originating in different solar position angles, Solar Phys., 249, 369-380, 2008c. 
Melendez, J. L. M., Sawant, H. S., Fernandes, F. C. R., and Benz, A. O.: Statistical analysis of high-frequency decimetric type III bursts, Solar Phys., 187, 77-88, 1999.

Storini, M., Bazilevskaya, G. A., Fliickiger, E. O., Krainev, M. B., Makhmutov, V. S., and Sladkova, A. I.: The GNEVYSHEV gap: A review for space weather, Adv. Space Res., 31, 895-900, 2003.
Wang, Y. M. and Sheeley, N. R.: Sunspot activity and the longterm variation of the Sun's open magnetic flux, J. Geophys. Res., 107(A10), 1302, doi:10.1029/2001JA000500, 2002.

Wang, Y. M., Lean, I., and Sheeley Jr., N. R.: The long-term variation of the Sun's open magnetic flux, Geophys. Res. Lett., 27, 505-508, 2000. 